\title{
Light-meson properties from the Bethe-Salpeter equation
}

\author{
M. Blank and A. Krassnigg \\ Institut für Physik, Karl-Franzens-Universität Graz, A-8010 Graz, Austria
}

\begin{abstract}
We discuss how to extract observables from an inhomogeneous vertex Bethe-Salpeter equation without resorting to the corresponding homogeneous equation. As an example we present a prediction for the $e^{+} e^{-}$decay width of the $\rho(1450)$ or $\rho^{\prime}$ meson. We also attempt to identify the momentum range contributing to a vector meson's decay constant.
\end{abstract}

Keywords: inhomogeneous Bethe-Salpeter equation, vector mesons, decay constants PACS: $14.40 .-\mathrm{n}, 13.20 .-\mathrm{v}, 12.38 . \mathrm{Lg}, 11.10 . \mathrm{St}$

\section{INTRODUCTION}

Due to their quantum numbers, vector-meson resonances are easily produced in $e^{+} e^{-}$scattering. In addition, the $\rho$ is among the lightest mesons and thus object of numerous studies and a prime target for theoretical investigation. In QCD, the Dyson-Schwinger-equation approach offers a nonperturbative continuum method to study mesons as bound states of quarks and gluons via the Bethe-Salpeter equation. Herein we present some new results in the light of recent progress regarding the methods used to approach such a bound-state problem.

\section{THE VERTEX BSE}

A general vertex $\Gamma(q, P)$, the inhomogeneous BetheSalpeter amplitude (iBSA), that connects quark and antiquark to a color-singlet current satisfies the equation

$$
\Gamma(p, P)=\Gamma_{0}+\int \frac{d^{4} q}{(2 \pi)^{4}} K(P, p, q) S_{a}\left(q_{+}\right) \Gamma(q, P) S_{b}\left(q_{-}\right)
$$

(inhomogeneous or vertex BSE), where $K$ is the quarkantiquark scattering kernel, and $\Gamma_{0}$ a current which defines the channel under investigation by spin, parity and charge-conjugation parity. In this work, we investigate vector quantum numbers, $J^{P C}=1^{--}$, such that we choose (cf. [1])

$$
\Gamma_{0}=Z_{2} \gamma_{\mu}
$$

where the renormalization constant $Z_{2}$ is calculated from the gap equation, cf. [2].

The iBSA has poles at the positions of the respective bound states, and it can be decomposed as

$$
\Gamma^{\mu}(q, P)=\sum_{i} \mathscr{N}_{i} \frac{\Gamma_{[h]}^{\mu}\left(q, P_{i}\right)}{P^{2}-P_{i}^{2}}+\text { regular terms },
$$

where $\Gamma_{[h]}$ is the homogeneous BSA of the meson under investigation, $P_{i}^{2}=-M_{i}^{2}$ the total momentum squared of the excitation $i$ in the respective channel, and $\mathscr{N}_{i}$ denotes a normalization constant.

We work in the well-established setup of the rainbowladder truncation and the effective interaction proposed by Maris and Tandy [3], with light-quark masses and the parameter $\omega=0.4 \mathrm{GeV}$ (except where noted), as given in [4].

\section{MASSES AND DECAY CONSTANTS}

According to Eq. (3), each bound state results in a pole in the iBSA $\Gamma^{\mu}(P, q)$. As described in [5], the amplitude is decomposed into components $F_{i}\left(P^{2}, q^{2}, P \cdot q\right)$ and covariants $T_{\mu}^{i}(P, q, \gamma)$ according to

$$
\Gamma^{\mu}\left(P^{2}, q^{2}, P \cdot q\right)=\sum_{i} F_{i}\left(P^{2}, q^{2}, P \cdot q\right) T_{\mu}^{i}(P, q, \gamma),
$$

where the standard vector covariants [4] are orthonormalized according to $\operatorname{Tr}\left[T_{\mu}^{i}(P, q, \gamma) T_{\mu}^{j}(P, q, \gamma)\right]=\delta_{i j}$. It is well-known how to obtain meson masses and decay constants using the corresponding homogeneous BSE. Here, however, we explore a different approach.

We calculate the bound state masses by fitting the inverse of the first component $F_{1}\left(P^{2}, 0,0\right)$, as shown in Fig. 1. We obtain

$$
m_{\rho}=0.774 \mathrm{GeV} \quad m_{\rho^{\prime}}=1.034 \mathrm{GeV}
$$

which is in agreement with [4] and [6].

The decay constant for a vector meson is given by [3]

$M_{i} f_{v}\left(P_{i}^{2}\right)=\frac{Z_{2}}{3} \int \frac{d^{4} q}{(2 \pi)^{4}} \operatorname{Tr}\left[\gamma_{\mu} S_{a}\left(q_{+}\right) \Gamma_{[h]}^{\mu}\left(q, P_{i}\right) S_{b}\left(q_{-}\right)\right]$,

where the trace runs over color and Dirac-indices. To extract the same information from the iBSA, we first consider the general case of a projection $f_{\tilde{\Gamma}}^{(i h)}\left(P^{2}\right)$ of the 


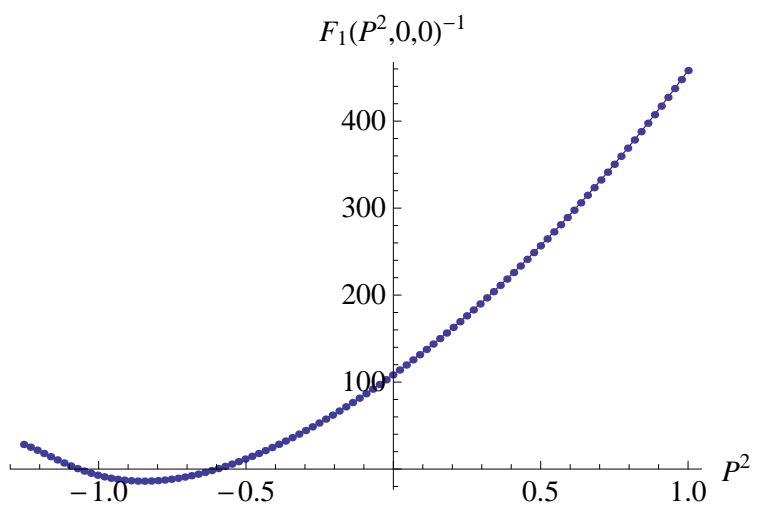

FIGURE 1. The inverse of the first component of the inhomogeneous vector amplitude $1 / F_{1}\left(P^{2}, 0,0\right)$, as a function of the square of the total momentum $P^{2}$. The zero-crossings give the masses of the ground state and the first excitation.

iBSA $\Gamma(q, P)$ on a current $\tilde{\Gamma}$,

$$
f_{\tilde{\Gamma}}^{(i h)}\left(P^{2}\right)=\int \frac{d^{4} q}{(2 \pi)^{4}} \operatorname{Tr}\left[\tilde{\Gamma} S_{a}\left(q_{+}\right) \Gamma(q, P) S_{b}\left(q_{-}\right)\right] .
$$

The poles in $\Gamma(q, P)$ translate into poles of $f_{\tilde{\Gamma}}^{(i h)}\left(P^{2}\right)$. In order to calculate the corresponding on-shell projection $f_{\tilde{\Gamma}}\left(P^{2}\right)$ (which ultimately gives the decay constant), the inhomogeneous BSE is rewritten as [1]

$$
\Gamma(p, P)=\Gamma_{0}+\int \frac{d^{4} q}{(2 \pi)^{4}} M(P, p, q) S_{a}\left(q_{+}\right) \Gamma_{0} S_{b}\left(q_{-}\right) .
$$

$M(P, p, q)$ denotes the fully amputated quark-antiquark scattering matrix which contains the bound state poles and may therefore be written as $[1,7]$

$$
M(P, p, q)=\sum_{i} \frac{\Gamma_{[h]}\left(p, P_{i}\right) \bar{\Gamma}_{[h]}\left(q,-P_{i}\right)}{P^{2}-P_{i}^{2}}+\text { reg. terms },
$$

with the index $i$ labeling the bound states in this channel, and $\bar{\Gamma}_{[h]}$ denoting the charge conjugation of $\Gamma_{[h]}$ (see, e.g., [2]). Inserting Eqs. (8) and (9) in Eq. (7), we find

$$
\begin{aligned}
& \quad f_{\tilde{\Gamma}}^{(i h)}\left(P^{2}\right)= \\
& \quad \sum_{i} \int \frac{d^{4} p d^{4} q}{(2 \pi)^{8}} \operatorname{Tr}\left[\tilde{\Gamma} S_{a}\left(p_{+}\right) \Gamma_{[h]}\left(p, P_{i}\right) S_{b}\left(p_{-}\right)\right] \\
& \times \frac{\operatorname{Tr}\left[\bar{\Gamma}_{[h]}\left(q,-P_{i}\right) S_{a}\left(q_{+}\right) \Gamma_{0} S_{b}\left(q_{-}\right)\right]}{P^{2}-P_{i}^{2}}+\text { reg. terms. }
\end{aligned}
$$

Thus, in order to obtain $f_{\tilde{\Gamma}}\left(P_{i}^{2}\right)$, one has to divide the residue of $f_{\tilde{\Gamma}}^{(i h)}\left(P^{2}\right)$ by the square root of the corresponding residue of the projection on the inhomogeneous term, $f_{\Gamma_{0}}^{(i h)}\left(P^{2}\right)$.

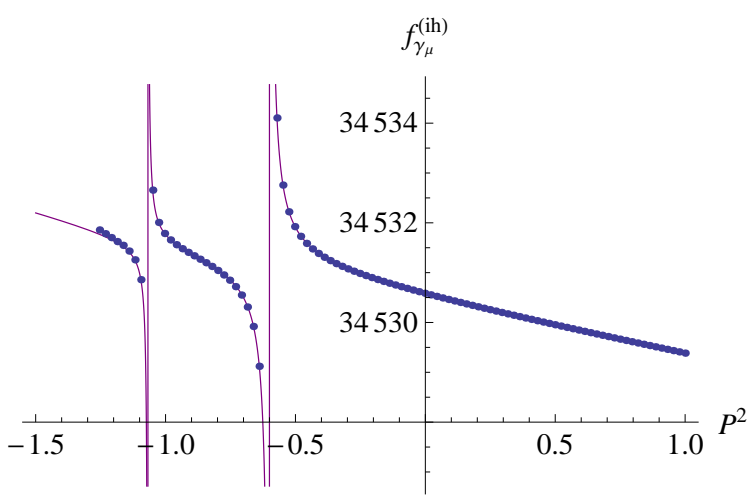

FIGURE 2. The projection $f_{\gamma_{\mu}}^{(i h)}\left(P^{2}\right)$ defined in Eq. (7). The line represents a pole fit to the data points, where the pole positions were taken from the fit of Fig. 1.

In the case of $f_{v}\left(P_{i}^{2}\right)$ the inhomogeneous term and the current are identical, $\Gamma_{0} \equiv \tilde{\Gamma}=Z_{2} \gamma_{\mu}$. The decay constants can therefore be calculated using only the residues $r_{i}$ of $f_{\gamma_{\mu}}^{(i h)}\left(P^{2}\right)$, which are extracted from the pole fit shown in Fig. 2, where the masses (5) are used as input. Comparing Eqs. (10) and (6), we find

$$
f_{v}\left(P_{i}^{2}\right)=\sqrt{\frac{r_{i}}{3\left(-P_{i}^{2}\right)}} .
$$

\section{PREDICTION FOR $f_{\rho^{\prime}}$}

The resulting decay constants of the $\rho$-meson and its first radial excitation corresponding to the masses in (5) are

$$
f_{\rho}=0.213 \mathrm{GeV} \quad f_{\rho^{\prime}}=0.079 \mathrm{GeV},
$$

which perfectly agrees with the results from the corresponding homogeneous BSE. To arrive at a concrete prediction for the decay width of the $\rho^{\prime}$ into $e^{+} e^{-}$we need to investigate the sensitivity of these results to the characteristic parameter of the model of Ref. [3]. Indeed, the excited-state result exhibits a considerable dependence, while for the ground state the results for both $m$ and $f$ are rather solid, i.e., a dependence is observed but small (of the order of $2 \%$ for $m$ and $7 \%$ for $f$, see $[4,8,9]$ for in-depth information and a thorough discussion)

Here the important point is that one can use the systematic behavior of this dependence to determine both a preferred parameter value or range for an ideal description in a phenomenological sense as well as to provide an estimate of the systematic effects in the calculation. As 'best parameter' in our case we extract $\omega=0.5 \mathrm{GeV}$ and obtain

$$
m_{\rho}=0.762 \mathrm{GeV} \quad f_{\rho}=0.218 \mathrm{GeV},
$$


for the ground state, which translates into a width $\Gamma_{e^{+} e^{-}}$ of $6.92 \mathrm{keV}$. The PDG [10] quotes an experimental value of $7.04 \pm 0.06 \mathrm{keV}$. For the $\rho^{\prime}$ the experimental situation is less clear: The PDG quote but don't use two results of the order of and smaller than $0.1 \mathrm{keV}$. Our excited-state result corresponding to (13) lies in a range accessible to us only via extrapolation techniques due to the analytic structure of the quark propagator (see, e.g., [11] for a discussion). However, we use extrapolations both in model-parameter and momentum space to reduce the uncertainty. We find for $\rho^{\prime}$

$$
f_{\rho^{\prime}}=0.095 \pm 0.039 \mathrm{GeV} \quad \Gamma_{e^{+} e^{-}}=0.94 \pm 0.66 \mathrm{keV} .
$$

\section{DISASSEMBLING $f_{\rho}$}

To obtain further information on the structure of the $\rho$-meson and its radial excitation, the contributions to the decay constants from the different momentum scales are investigated. Therefore, we solve in addition to the inhomogeneous also the homogeneous BSE, from which the decay constant can be calculated according to Eq. (6), and define the density $d_{f_{v}}\left(p^{2}\right)$ via

$$
f_{v}\left(P_{i}^{2}\right)=\left(1 / \sqrt{-P_{i}^{2}}\right) \int d p^{2} d_{f_{v}}\left(p^{2}\right) .
$$

$d_{f_{v}}\left(p^{2}\right)$ is plotted in Fig. 3 for the ground state and first radial excitation of the $\rho$-meson. The main contributions are centered in the mid-momentum regime around $p^{2}=0.1 \mathrm{GeV}^{2}$, and neither the UV nor the IR have a strong influence. This supports [12], where it was shown that $f_{\rho}$ (among other quantities) is insensitive to the behavior of the effective interaction in the far infrared. The same is true for the (perturbatively determined) UV domain. Therefore, an effective interaction, which neglects this, quite naturally gives reliable results for the decay constant as well, cf. [13].

We further note that the scale dependence of analogous projections has also been investigated in lattice QCD [14].

\section{CONCLUSIONS}

We have demonstrated how the masses and decay constants of the $\rho$ meson and its radial excitation can be calculated from the inhomogeneous (vertex) Bethe-Salpeter equation without using any information from the corresponding homogeneous solutions, which is in contrast to a previous study [15]. We have exemplified our method via a reasonable result for $f_{\rho^{\prime}}$.

Furthermore, we have investigated the contributions to $f_{\rho}$ and $f_{\rho^{\prime}}$ from different momentum scales, and found

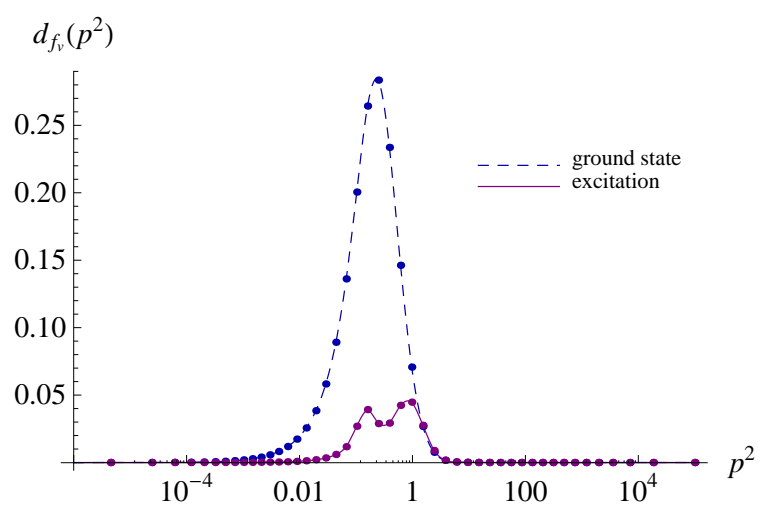

FIGURE 3. Plot of the density $d_{f_{v}}\left(p^{2}\right)$ for the vector ground and excited states.

significant contributions from neither the far IR nor the UV domain.

\section{ACKNOWLEDGMENTS}

We would like to acknowledge valuable discussions with R. Alkofer, G. Eichmann, D. Horvatić, C. B. Lang, M. Limmer, and V. Mader. This work was supported by the Austrian Science Fund $F W F$ under project no. P20496-N16, and was performed in association with and supported in part by the $F W F$ doctoral program no. W1203-N08.

\section{REFERENCES}

1. P. Maris, and P. C. Tandy, Phys. Rev. C 61, 045202 (2000).

2. P. Maris, and C. D. Roberts, Phys. Rev. C 56, 3369-3383 (1997).

3. P. Maris, and P. C. Tandy, Phys. Rev. C 60, 055214 (1999).

4. A. Krassnigg, Phys. Rev. D 80, 114010 (2009).

5. M. Blank, and A. Krassnigg (2010), arXiv:1009.1535.

6. A. Krassnigg, PoS Confinement8, 75 (2009).

7. P. Maris, C. D. Roberts, and P. C. Tandy, Phys. Lett. B 420, 267-273 (1998).

8. A. Höll, A. Krassnigg, and C. D. Roberts, Phys. Rev. C 70, 042203(R) (2004).

9. A. Höll, A. Krassnigg, P. Maris, C. D. Roberts, and S. V. Wright, Phys. Rev. C 71, 065204 (2005).

10. K. Nakamura, et al., J. Phys. G 37, 075021 (2010).

11. M. Bhagwat, M. A. Pichowsky, and P. C. Tandy, Phys. Rev. D 67, 054019 (2003).

12. M. Blank, A. Krassnigg, and A. Maas (2010), arXiv:1007.3901.

13. R. Alkofer, P. Watson, and H. Weigel, Phys. Rev. D 65, 094026 (2002).

14. L. Y. Glozman, C. B. Lang, and M. Limmer, PoS LATTICE2010, 149 (2010).

15. M. S. Bhagwat, A. Höll, A. Krassnigg, C. D. Roberts, and S. V. Wright, Few-Body Syst. 40, 209-235 (2007). 